\title{
AN ANALYSIS OF VARIOUS MACHINE LEARNING ALGORITHMS AND THEIR IMPLEMENTATIONS IN THE CONTEMPORARY WORLD
}

\author{
Malav Patel $^{1}$, Abhi Priyadarshi ${ }^{2}$ \\ Student, Adani Institute of Infrastructure Engineering, Ahmedabad, Gujarat ${ }^{1,2}$ \\ patelmalav5265@gmail.com ${ }^{l}$ \\ abhipriyadarshi28@gmail.com ${ }^{2}$ \\ $* * *$
}

\begin{abstract}
Machine Learning is the logical investigation of calculations and measurable models that PC frameworks use to play out a particular undertaking without being unequivocally modified. Machine Learning (ML) uses calculations in numerous applications that we utilize every day. The machine learning algorithms are developed in a way that they acquire information from experience and their performance improves as they digest on more and more data. Each time a web index like Google is utilized to look through the web, one reason that it functions admirably is on the grounds that a learning calculation has figured out how to display relevant site pages. This paper will touch base the different machine learning apparatus which are expected to run the machine learning ventures and furthermore where these instruments are actualized.
\end{abstract}

Keywords: Machine Learning, Statistical, Programmed, Algorithm

\section{INTRODUCTION}

Machine Learning uses information mining techniques to extract the data from the huge sized datasets. ML and Data Mining techniques explore information from end to end to locate the hidden patterns inside the dataset [1]. Machine learning is a multidisciplinary field having a wide-range of research spaces reinforcing its existence. The recreation of ML models is essentially related to Computational Statistics whose primary point is to zero in on making predictions through computers. It is additionally co-related to Mathematical Optimization which relates models, applications and frameworks to the field of measurements. Real world problems have high complexity which makes them excellent candidates for utilization of ML [2].

Machine learning (ML) is used to teach machines how to handle the information more efficiently. Sometimes after viewing the information, we can't interpret the extracted data from the information. In that case, we apply machine learning. With the abundance of datasets available, the demand for machine learning is on a rise [3]. Machine Learning relies on different algorithms to solve information problems. Information scientists like to bring up that there's no single "one-size-fits-all" type of algorithm that is best to solve a problem [4]. The type of algorithm employed depends on the category of the problem you wish to solve, the number of variables, the sort of model that would suit it best, etc.

\section{LITERATURE REVIEW}

George Tzanis (2006) [5] Machine learning is one of the older areas of man-made consciousness and concerns the investigation of computational methods for the discovery of new knowledge and for the management of existing knowledge. Machine learning methods have been applied to different application domains. However, a few years ago due to different technological advances and research efforts (e.g. completion of the Human Genome Project, evolution of the Web), new data have been available and consequently new domains where machine learning can be applied have arisen. Some of these modern applications are learning from biological sequences, learning from email data, and learning in complex environments, for example, Web. This paper presented the above three application domains just as some 


\section{|| Volume 5 || Issue 12 || December 2020 || ISSN (Online) 2456-0774 INTERNATIONAL JOURNAL OF ADVANCE SCIENTIFIC RESEARCH}

\section{AND ENGINEERING TRENDS}

recent efforts, where machine learning techniques are applied to analyze the data provided by these domains.

Francesco Musumeci (2018) [6] The present telecommunication networks have become sources of enormous measures of widely heterogeneous data. This data can be retrieved from network traffic traces, network alerts, signal quality markers, users' behavioral data, etc. Advanced mathematical tools are required to extract meaningful information from these data and take decisions pertaining to the proper working of the networks from the networkgenerated data. Among these mathematical tools, Machine Learning (ML) is regarded as one of the most encouraging methodological approaches to perform network-data examination and enable automated network self-setup and flaw management. The selection of ML techniques in the field of optical correspondence networks is motivated by the unprecedented development of network complexity faced by optical networks over the most recent couple of years. Such increase in complexity is due to the presentation of a huge number of adjustable and interdependent system parameters that are enabled by the usage of coherent transmission/reception technologies, advanced computerized signal processing and compensation of nonlinear effects in optical fiber proliferation. In this paper we provide an overview of the utilization of ML to optical correspondences and networking. We classify and survey relevant literature dealing with the point, and we additionally provide a basic instructional exercise on ML for researchers and practitioners interested in this field. Although a decent number of research papers have recently appeared, the utilization of ML to optical networks is still in its outset: to stimulate further work in this area, we conclude the paper proposing new possible research directions.

Rekha Nagar (2019) [7] Machine Learning (ML) has unfurled from Artificial Intelligence, a field of computer science. Machine Learning (ML) is a multidisciplinary field ,a blend of measurements and computer science algorithms which is widely used in predictive analyses and classification. The second section of the paper center to influence the basic machine learning methods and algorithms. This paper will experience the different machine learning tools needed to run the machine learning projects. The primary concern of the paper is the examination of the principle approaches and case studies of utilizing machine learning for forecasting in different areas such stock price forecasting, the travel industry demand forecasting, sun oriented light forecasting, supply chain demand and consideration of neural networks in machine learning methods.

Jafar Alzubi (2019) [8] The current SMAC (Social, Mobile, Analytic, Cloud) technology trend paves the route to a future wherein intelligent machines, networked processes and big data are united. This virtual world has generated immense measure of data which is accelerating the reception of machine learning solutions and practices. Machine Learning enables computers to imitate and adjust human-like behavior. Utilizing machine learning, each interaction, each activity performed, becomes something that the system can learn and use as an experience for the next time. This work is an overview of this data examination method which enables computers to learn and do what comes normally to people, i.e. learn for a fact. It includes the preliminaries of machine learning, the definition, nomenclature and the applications; describing it's what, how and why. The technological guide of machine learning is discussed to understand and verify its potential as a market and industry practice. The essential intent of this work is to give knowledge into why machine learning is the future.

\section{APPLICATIONS OF MACHINE LEARNING}

Machine learning has been extensively applied in different application domains. Some of the most well known applications include medical conclusion, credit hazard examination, customer profiling, market segmentation, targeted marketing, retail management and misrepresentation detection [9]. In the most recent years due to different technological advances and research efforts like the completion of the Human Genome Project and the evolution of the Web, new data have been available and consequently new domains where machine learning can be applied have arisen. Some of these modern applications are Medical decision making, Optimized gaming, Medical imaging, Autonomous delivery, etc.

\section{MACHINE LEARNING AND HUMAN LEARNING}

A research area closely related to Machine Learning is the investigation of the human and creature mind in Neuroscience, Psychology, and related fields. The researchers proposed that how a machine could learn as a matter of fact most likely would not be altogether different than how a creature or a human psyche learns with time and experience. However, the research concentrated on tackling machine learning problems utilizing learning methods of the 


\section{|| Volume 5 || Issue 12 || December 2020 || ISSN (Online) 2456-0774 INTERNATIONAL JOURNAL OF ADVANCE SCIENTIFIC RESEARCH}

\section{AND ENGINEERING TRENDS}

human mind didn't yield a lot of promising results so far than the research concerned with statistical - computational methodology. This may be due to the way that the human or creature brain research remains not completely understandable to date [10]. Regardless of these difficulties, cooperation between human learning and machine learning is increasing; for machine learning is being used to explain several learning techniques seen in both, humans and creatures. For example, a machine learning method of temporal difference was proposed to explain neural signals in creature learning. It is genuinely expected that this joint effort is to fill considerably in coming years.

\section{CLASSIFICATION OF MACHINE LEARNING METHODS}

Rule Induction: Rule induction is to produce a decision tree or a set of decision rules from the preparation set to classify. The primary advantage of rule induction is that it has a solid capacity to process large data sets and is suitable for classification and predictive errands. The results are easy to interpret and technically easy to implement.

Neural Networks: The neural network consists of processing nodes similar to neurons in the human brain. The info node is connected to the yield node through a set of hidden nodes to frame a multi-layer network structure. The neural network learns through repeated networks preparing on authentic sample data. The greatest advantage of a neural network is that it can accurately predict complex problems.

Case reasoning: Each case consists of two sections: problem description and answer for the problem. After posting inquiries, the system will search for coordinating cases and arrangements. Its advantage is that it can better deal with contaminated and missing data, which is very suitable for some cases.

Genetic algorithm: Genetic algorithm is a combinatorial improvement method based on biological evolution processes. Genetic search is based on the Darwinian Theory of endurance of fittest. The basic idea is natural selection and the best or better person. The operation process includes reproduction, hybridization and change. The advantage of genetic algorithms is that it is easy to integrate with other systems.

Inductive logic programming: Inductive logic programming uses first-level attribute logic to define and describe concepts. Where this technique has a solid conceptual description mechanism to express complex relations. To start with, it defines positive and negative examples and afterwards it ranks the new examples. From inductive machine learning, ILP inherits its goal: to develop tools and techniques to induce hypotheses from observations (examples) and to synthesize new knowledge from experience [11] It exerts a consistent populace of people to search a sample of space. Every one of the populace is evaluated by fitness.

\section{TYPES OF ALGORITHM}

Decision Tree: Decision Tree is a technique for approximating discrete valued target work which represents the learnt work as a decision tree [12]. A decision tree classifies instances by arranging them from root to some leaf nodes based on feature values. Each node represents some decision (test condition) on the attribute of the instance whereas every branch represents a possible value for that feature. Classification of an instance begins at the root node called the decision node. Based on the value of the node, the tree traverses down along the edge which corresponds to the value of the yield of the feature test. This process continues in the sub-tree headed by the new node toward the end of the previous edge. At last, the leaf node signifies the classification categories or an official conclusion. While utilizing a decision tree, center is around how to decide which attribute is the best classifier at each node level. Statistical measures like data gain, Gini index, Chi-square and entropy are calculated for each node to calculate the value of that node. Several algorithms are used to implement decision trees. The most mainstream ones are: Classification and Regression Tree (CART), Iterative Dichotomiser 3 (ID3), Automatic Interaction Detection (CHAID), ChiSquared C4.5 and C5.0 and M5.

Naïve Bayes: Naïve Bayes classifies utilizing Bayes' Theorem of likelihood. The naive Bayes classifier greatly simplifies learning by assuming that features are independent given class [13]. Naïve Bayes' classifiers fall under the category of simple probabilistic classifiers based on the concept of Bayes' Theorem having solid independence assumptions among the features. It is especially suited when the dimensionality of the sources of info is high.

Support Vector Machines: SVMs can be used for classification just as regression problems. It is a supervised learning algorithm. It deals with the concept of edge count. In this algorithm, each data item is plotted as a point in ndimensional space. The value of each feature is the value of 


\section{|| Volume 5 || Issue 12 || December 2020 || ISSN (Online) 2456-0774 INTERNATIONAL JOURNAL OF ADVANCE SCIENTIFIC RESEARCH}

\section{AND ENGINEERING TRENDS}

the corresponding coordinate. It classifies the data into different classes by finding a line which separates the training datasets into classes. It works by boosting the distances between the nearest data point and the hyperplane that we can call as edge [14].

Regression Analysis: Regression examination is a predictive modeling technique which investigates the relationship between a dependent (target) and independent variable(s) (predictor). It is a significant device for dissecting and modeling of data. In this method, we attempt to fit the line/curve to the data focuses to minimize the differences between distances of data focuses from the curve or line. There are different sorts of regression investigation like linear, calculated and polynomial. The regression using one single independent variable is called univariate regression analysis while the analysis using more than one independent variable is called multivariate regression analysis.[15]

K-Means Clustering: K-means [16] is a well-known unsupervised machine learning algorithm for cluster examination. It will probably segment ' $n$ ' observations into ' $k$ ' clusters in which each observation belong to the cluster having the nearest mean, serving as a prototype of the cluster. The mean of the observations in a specific cluster defines the center of the cluster.

\section{IMPLEMENTATION OF MACHINE LEARNING}

Commute Estimation: In general, a single excursion takes more than average time to complete, multiple modes of transportation are used for an outing including traffic timing to reach the destination. Additionally, the results revealed from predictions based on machine learning algorithms and urban indicators by Gabriel Spandon [17] and team can reconstruct the commuter's network with $90.4 \%$ of accuracy and describe the $77.6 \%$ of the variance which is observed in the flow of people between cities.

Google's Map: Utilizing the area data from smartphones, Google Maps can inspect the dexterity of moving traffic anytime, moreover Google guide can also organize userreported traffic like traffic development and accidents. By accessing relevant data and appropriate fed algorithms, Google Maps can reduce driving time by showing the fastest route [18].

Riding Apps: ML helps the organization to estimate the price of a ride, registering an ideal pickup area and ensuring the shortest route of the outing. The universal accessibility of the Internet and progressed computing frameworks have brought about the rapid development of keen urban communities. From associated gadgets to live vehicle following, technology is taking the field of transportation to another level. A fundamental piece of the transportation domain in keen urban communities is Ride Sharing.

Commercial flights to use Autopilot: With the help of AI technology, Autopilots are take care of flights now. In a report of The NewYork Times, pilots reported doing manual flying of seven minutes, primarily during takeoff and landing, and the rest of the flying is done via autopilots.

Spam Filters: Some rules-based filters aren't served actively in an email inbox, for example, when a message comes with the words "online consultancy", "online drug store", or from "obscure address". ML is offering a powerful feature that filters email from a variety of signals, like words in the message, metadata of the message. However, it even filters the emails based on "everyday deals" or "welcome messages" etc. With the use of ML, Gmail filters $99.9 \%$ of spam messages [19].

Smart Replies: It is a novel end-to-end method for automatically generating short email responses, called Smart Reply. It generates semantically diverse suggestions that can be used as complete email responses with just one tap on mobile.[20]

Playing Checkers Game: A computer program learns how to play checkers game, improvises its performance as determined by its capacity to succeed at different classes of undertakings including the game, through experience obtained by messing around against itself.

Speech Recognition: The most sophisticated speech recognition systems these days deploy machine learning algorithms in some structures. Example: SPHINX system learns speaker-specific sounds and words from speech signals. Different Neural Network learning methodologies for interpreting hidden Markov Models are exceptionally effective for consequently altering speakers, word reference, noise etc.

Computer Vision: Majority of recent computer vision systems like facial recognition software, systems capable of programmed classification of minuscule images of cells employ machine learning approaches for better exactness. For example, the US Post Office uses a computer vision system with a penmanship analyzer in this way trained to sort letters with handwritten addresses naturally with precision levels as high as $85 \%$ [21]. 


\section{|| Volume 5 || Issue 12 || December 2020 || ISSN (Online) 2456-0774 INTERNATIONAL JOURNAL OF ADVANCE SCIENTIFIC RESEARCH}

\section{AND ENGINEERING TRENDS}

Explaining Human Learning: As mentioned earlier, machine learning theories have been perceived fitting to comprehend features of learning in people and creatures. Reinforcement learning algorithms estimate the dopaminergic neurons induced activities in creatures during reward-based learning with astounding precision. ML algorithms are used for uncovering irregular delineations of normally appearing images and predict the visual features detected in creatures' underlying visual cortex. Nevertheless, the significant drivers in human or creature learning like incitement, frightfulness, urgency, hunger, instinctive activities and learning by experimentation over numerous time scales are not yet taken into account in ML algorithms. This is a potential occasion to discover a more generalized concept of learning that entails the two: creatures and machines.

\section{CONCLUSION}

Machine Learning can be Supervised or Unsupervised. On the off chance that you have lesser measure of data and clearly labeled data for training, pick Supervised Learning whereas Unsupervised Learning would generally give better performance and results for large data sets. On the other hand where you have a huge data set easily available, dive for deep learning techniques. The machine learning mechanism in data mining has been basically implemented in numerous industries and associations, including telecommunication industry, banking sector, money related industry, retail industry, insurance industry, etc. For example, in the money related industry, monetary examiners use data mining to fabricate prediction models to identify the patterns that have caused market instability over the years, thereby improving the capacity of predicting market unpredictability. Machine learning acts as a key technology driver that encompasses the intelligent power to harness the knowledge from the available data.

\section{REFERENCES}

1.S. R. Guruvayur and R. Suchithra, "A detailed study on machine learning techniques for data mining," 2017 International Conference on Trends in Electronics and Informatics (ICEI), Tirunelveli, 2017, pp. 1187-1192.

2.P. Ongsulee, "Artificial intelligence, machine learning and deep learning," 2017 15th International Conference on ICT and Knowledge Engineering (ICT\&KE), Bangkok, 2017, pp. $1-6$.
3.M. Xue and C. Zhu, "A Study and Application on Machine Learning of Artificial Intelligence," 2009 International Joint Conference on Artificial Intelligence, Hainan Island, 2009, pp. 272-274.

4. Q. Zhou and Z. Huang, "A Decision-Making Method Using Knowledge-Based Machine Learning," 2012 International Conference on Computer Science and Electronics Engineering, Hangzhou, 2012, pp. 616-620.

5. George Tzanis, Modern Applications of Machine Learning, Proceedings of the 1st Annual SEERC Doctoral Student Conference - DSC 2006

6. Francesco Musumeci, (2018), An Overview on Application of Machine Learning Techniques in Optical Networks, arXiv:1803.07976v4 [cs.NI] 1 Dec 2018

7. Rekha Nagar, (2019), A literature survey on Machine Learning Algorithms, JETIR, Volume 6, Issue 4

8. Alzubi, Jafar\&Nayyar, Anand\& Kumar, Akshi. (2018). Machine Learning from Theory to Algorithms: An Overview. Journal of Physics: Conference Series. 1142. 012012. 10.1088/1742-6596/1142/1/012012.

9.J. Vijaya, Chandra Narasimham, M. Ali Hussain, "Data and Information Storage Security from Advanced Persistent Attack in Cloud Computing", International Journal of Applied Engineering Research, 2014.

10.Chharia and R. K. Gupta, "Email classifier: An ensemble using probability and rules," 2013 Sixth International Conference on Contemporary Computing (IC3), Noida, 2013, pp. 130-136.

11. STEPHEN MUGGLETON AND LUC DE RAEDT, INDUCTIVE LOGIC PROGRAMMING: THEORY AND METHODS, J. LOGIC PROGRAMMING 1994: 19,20:629679

12.J. Vijaya Chandra, (2019), A Framework for Implementing Machine Learning algorithms using Data sets, International Journal of Innovative Technology and Exploring Engineering (IJITEE), Volume-8 Issue-11

13.I.Rish, "An empirical study of the naive Bayes classifier," IJCAI workshop on Empirical Methods in AI, 2005.

14.T. Vyas, P. Prajapati and S. Gadhwal, "A survey and evaluation of supervised machine learning techniques for spam e-mail filtering," 2015 IEEE International Conference on Electrical, Computer and Communication Technologies (ICECCT), Coimbatore, 2015, pp. 1-7. 
15.Tabachnick, B. G., \& Fidell, L. S. (1996). Using multivariate statistics. New York: HarperCollins College Publishers.

16.J. MacQueen, "Some Methods for Classification and Analysis of Multivariate Observations," in 5th Berkeley Symposium on Mathematical Statistics and Probability, 1964.

17.A. C. P. L. f. d. C. f. R.-J. L. G. A. A. Gabriel Spadon, "Reconstructing commuters network using machine learning and urban indicators," Nature, 2019.

18.AdwanYasin and Addelmunem Abuhasan, "AN INTELLIGENT CLASSIFICATION MODEL FOR PHISHING EMAIL DETECTION" , International Journal of Network Security \& Its Applications (IJNSA), Vol.8, No.4,July 2016.

19.. R. Islam, J. Singh, A. Chonka and W. Zhou, "Multiclassifier Classification of Spam Email on a Ubiquitous Multi-Core Architecture," 2008 IFIP International Conference on Network and Parallel Computing, Shanghai, 2008, pp. 210-217.

20.Young, Peter, and Vivek Ramavajjala. "Smart Reply: Automated Response Suggestion for Email." KDD '16: Proceedings of the 22nd ACM SIGKDD International Conference on Knowledge Discovery and Data Mining, 2016, pp. 955-964

21.Shokri R, Stronati M, Song C, Shmatikov V. Membership inference attacks against machine learning models. In Security and Privacy (SP), 2017 IEEE Symposium on 2017 May 22 (pp. 3-18). IEEE. 